\title{
AN EXISTENCE THEOREM FOR GENERALIZED PFAFFIAN SYSTEMS*
}

\author{
BY J. M. THOMAS
}

1. Introduction. The present paper has for its object the extension of Cartan's existence theorem $†$ for a pfaffian system to a system of equations whose left members are symbolic differential forms of arbitrary degrees. Although the proof of the general case involves no essentially new principle, the arrangement of the details given here appears simpler than in any proof previously published for the linear case $\ddagger$ and at the same time rigorous. The manner of presentation makes clear the relationship of the general theorem (and, consequently, of Cartan's theorem) to the theory of systems of partial differential equations as developed by Riquier.

2. The Existence Theorem. The set of equations

$$
\omega^{\lambda}=0, \quad(\lambda=1,2, \cdots, \rho),
$$

in which $\omega^{\lambda}$ is a symbolic differential form of degree $p_{\lambda}$, $\left(0<p_{\lambda} \leqq n\right)$, will be called a generalized pfaffian system. $\S$ The ordinary pfaffian system is the special case in which every $\omega$ is linear.

For the sake of simplicity, consider only one form temporarily, and put

$$
\omega=a_{i_{1} i_{2}} \cdots i_{p} d x^{i_{1}} d x^{i_{2}} \ldots d x^{i_{p}},
$$

where the $a$ 's are functions of the $x$ 's and are skew-symmetric

* Presented to the Society, December 1, 1933.

† E. Cartan, Annales de l'École Normale, (3), vol. 18 (1901), pp. 241-311; E. Goursat, Problème de Pfaff, 1922, pp. 343-381.

$\ddagger$ C. Burstin, Recueil Mathématique de la Société Mathématique de Moscou, vol. 37 (1930), pp. 13-21, has recognized that the proof given by Goursat for the linear case can be materially simplified. His proof, which is apparently open to objections, will be discussed in a subsequent note in connection with singular integral varieties.

$\S$ If the proposed system contains a set $S_{0}$ of equations whose left members are of degree zero, elimination of some of the variables $x$ by means of $S_{0}$ gives a system of the type considered. 
in every pair of subscripts. Suppose the $x$ 's expressed in terms of $k$ parameters

$$
x^{i}=f^{i}\left(u^{1}, u^{2}, \cdots, u^{k}\right), \quad(i=1,2, \cdots, n),
$$

and put

$$
d x^{i}=\frac{\partial x^{i}}{\partial u^{\alpha}} d u^{\alpha}=x_{\alpha}^{i} d u^{\alpha} .
$$

We then have

$$
\omega=\left[\alpha_{1} \alpha_{2} \cdots \alpha_{p}\right] d u^{\alpha_{1}} d u^{\alpha_{2}} \cdots d u^{\alpha_{p}},
$$

where

$$
\left[\alpha_{1} \alpha_{2} \cdots \alpha_{p}\right]=a_{i_{1} i_{2}} \cdots i_{p} x_{\alpha_{1}} x_{\alpha_{2}}^{i_{1}} i_{\alpha_{2}}^{i_{2}} \cdots x_{\alpha_{p}}^{i_{p}} .
$$

A similar notation is employed for the derived form:

$$
\omega^{\prime}=\left[\alpha_{1} \alpha_{2} \cdots \alpha_{p+1}\right]^{\prime} d u^{\alpha_{1}} d u^{\alpha_{2}} \cdots d u^{\alpha_{p+1}} .
$$

The following identity can then be proved:

$$
\begin{aligned}
& \frac{\partial\left[\alpha_{1} \cdots \alpha_{p}\right]}{\partial u^{\alpha_{p+1}}}+(-1)^{p} \frac{\partial\left[\alpha_{2} \cdots \alpha_{p+1}\right]}{\partial u^{\alpha_{1}}}+\cdots \\
& =(p+1)\left[\alpha_{1} \alpha_{2} \cdots \alpha_{p+1}\right]^{\prime},
\end{aligned}
$$

where the terms on the left are obtained by cyclic permutation of the indices on the first term and $(-1)^{p}$ occurs in the even numbered terms.

If substitution from (3) and (4) in $\omega=0$ gives identities in the $u$ 's and their differentials, (3) is called an integral variety of $\omega=0$. Its dimension is the character (see below) of the matrix

$$
J_{k}=\left\|x_{\alpha}^{i}\right\|, \quad(i=1,2, \cdots, n ; \alpha=1,2, \cdots, k) .
$$

The conditions that (3) be integral for $\omega=0$ are

$$
\left[\alpha_{1} \alpha_{2} \cdots \alpha_{p}\right]=0,
$$

where the $\alpha$ 's are given every set of $p$ distinct values chosen from $1,2, \cdots, k$.

From (8) and (7) we therefore see that the equation $\omega=0 \mathrm{im}$ plies $\omega^{\prime}=0$ in the sense that every integral variety of the former is an integral variety of the latter. 
A variety is integral for a system (1) provided it is integral for every equation in (1).

The system

$$
\omega^{\prime \lambda}=0,
$$

obtained by equating to zero the derived forms of the left members of (1), is also a generalized pfaffian system.* System (1) can be augmented by (11) without having its integral varieties altered. Since $\left(\omega^{\prime}\right)^{\prime} \equiv 0$, we then have to deal with a system (1) such that the derived form of every left member is either a left member or zero. We shall suppose, therefore, that every system (1) which we treat has been augmented so as to possess this property.

For convenience of treatment we divide the conditions that (3) be integral for (1) into sets $\Sigma$, the set $\Sigma_{\alpha}$ containing every condition (10) in which one of the indices is $\alpha$ and all the others less than $\alpha$. The equations of $\Sigma_{\alpha}$, for a fixed $\alpha$, are linear and homogeneous in the unknowns $x_{\alpha}{ }^{i}$. Let the matrix of $\Sigma_{\alpha}$ be $M_{\alpha}$.

A matrix $M$, whose elements are expressed in terms of certain variables, will be said to have character $m$ if every one of its determinants of order $m+1$ vanishes identically in all the variables and at least one determinant of order $m$ is different from zero for some values of the variables. A matrix is of rank $r$ on a variety (or in a region) if, when its elements are expressed in terms of the parameters of the variety, every determinant of order $r+1$ vanishes for all values of the parameters, and corresponding to every set of values for the parameters there is at least one determinant of order $r$ different from zero.

Denote the character of $M_{1}$ by $m_{1}$. Then $\Sigma_{1}$ can be solved for $m_{1}$ of the unknowns $x_{1}{ }^{i}$ in terms of the others. Let the results, substituted in $M_{2}$ and $\Sigma_{2}$, give $\bar{M}_{2}$ and $\bar{\Sigma}_{2}$. Denote the character of $\bar{M}_{2}$ by $m_{2}$. The number $m_{2}$ is the same for all general solutions of $\Sigma_{1}$.

Let the general solution of $\Sigma_{1}$ employed above and a similar general solution of $\bar{\Sigma}_{2}$ be substituted in $\Sigma_{3}, M_{3}$ to give $\bar{\Sigma}_{3}$ and $\bar{M}_{3}$, whose character is $m_{3}$. As before, the number $m_{3}$ does not depend on the mode of solution. Continue the process to form sequences $\bar{\Sigma}_{\alpha}, \bar{M}_{\alpha}, m_{\alpha}$.

Since we can solve $\Sigma_{1}, \cdots, \Sigma_{k}$ in the above manner for

* It might appropriately be called the derived system of (1), had not that name already been employed in another sense. 
$m_{1}, \cdots, m_{k}$ of the quantities $x_{1}{ }^{i}, \cdots, x_{k}{ }^{i}$, respectively, the totality of the quantities

$$
\left[\alpha_{1} \alpha_{2} \cdots \alpha_{p}\right], \quad\left(\alpha_{1}<\alpha_{2}<\cdots<\alpha_{p} \leqq k ; p \leqq k\right),
$$

formed for all $\omega$ 's in (1), can be taken with a properly chosen subset of

$$
x_{1}^{i}, \cdots, x_{k}^{i}
$$

to replace variables (13) in expressing $\left[\alpha_{1} \alpha_{2} \cdots \alpha_{p}(k+1)\right]$. Since that bracket reduces to $\left[\overline{\alpha_{1} \alpha_{2} \cdots \alpha_{p}(k+1)}\right]$ when (12) are zero and the subset of (13) has arbitrary values, Taylor's theorem gives

$$
\begin{aligned}
& {\left[\alpha_{1} \alpha_{2} \cdots \alpha_{p}(k+1)\right]} \\
& \quad=\left[\overline{\alpha_{1} \alpha_{2} \cdots \alpha_{p}(k+1)}\right]+\left\{\alpha_{1} \alpha_{2} \cdots \alpha_{p}(k+1)\right\},
\end{aligned}
$$

where the vanishing of (12) implies the identical vanishing of the braces \{\} .

Suppose that $x^{i}, x_{1}^{i}, \cdots, x_{\alpha}{ }^{i}$ satisfy systems $\Sigma_{1}, \cdots, \Sigma_{\alpha}$ and make the ranks of* $J_{\alpha}, M_{1}, \cdots, M_{\alpha+1}$, respectively, $\alpha, m_{1}, \cdots, m_{\alpha+1}$. Obviously the values $x_{\alpha+1}^{i}=x_{\beta}{ }^{i}$, where $\beta \leqq \alpha$, satisfy $\Sigma_{\alpha+1}$ and therefore $\bar{\Sigma}_{\alpha+1}$. Hence the number of independent solutions of $\bar{\Sigma}_{\alpha+1}$ is at least $\alpha$, that is,

$$
n-m_{\alpha+1} \geqq \alpha \text {. }
$$

If the inequality holds, we may repeat the argument with $\alpha+1$ replacing $\alpha$. Since $\alpha$ is increasing and $n-m_{\alpha+1}$ is non-increasing, we ultimately reach a value $\gamma$ such that $\dagger$

$$
n-m_{\gamma+1}=\gamma \text {. }
$$

This means that there is no set of solutions $x^{i}, x_{1}{ }^{i}, \cdots, x_{\gamma}{ }^{i}+1$ which makes the ranks of $J_{\gamma+1}, M_{1}, \cdots, M_{\gamma+1}$, respectively, $\gamma+1, m_{1}, \cdots, m_{\gamma+1}$. Consequently, the dimension $k$ of an integral variety on which each matrix $\bar{M}_{\alpha},(\alpha=1,2, \cdots, k+1$; $\left.\bar{M}_{1}=M\right)$, has rank equal to its character cannot exceed $\gamma$.

Let $k$ be a non-negative integer satisfying $k+1 \leqq \gamma$. Let the

* See formula (9).

$\dagger$ If we put $m_{\alpha}=s_{0}+\cdots+s_{\alpha-1}$, the $s$ 's are Cartan's characters in the linear case. 
coefficients of (1) be holomorphic in the neighborhood of a point

$$
\left(x^{i}\right)_{0}
$$

at which $M_{1}$ has rank $m_{1}$. Further, let variety (3) be a solution of $\Sigma_{1}, \cdots, \Sigma_{k}$ such that the $f$ 's are holomorphic in the neighborhood of

$$
u^{1}=\cdots=u^{k}=0,
$$

reduce to (16) at that point, and make the ranks of $J_{k}, M_{1}, \cdots$, $M_{k+1}$ respectively $k, m_{1}, \cdots, m_{k+1}$ in that neighborhood. The solved form of $\bar{\Sigma}_{k+1}$ is therefore valid in the neighborhood of the values (16) and

$$
\left(\frac{\partial f^{i}}{\partial u^{\alpha}}\right)_{u^{1}=\cdots=u^{k}=0}, \quad(\alpha=1,2, \cdots, k) .
$$

It is, moreover, a system to which Cauchy's theorem is applicable. We know, therefore, the existence of a set of functions

$$
x^{i}=\phi^{i}\left(u^{1}, \cdots, u^{k}, u^{k+1}\right)
$$

which satisfy system $\bar{\Sigma}_{k+1}$ and also

$$
\phi^{i}\left(u^{1}, \cdots, u^{k}, 0\right)=f^{i}\left(u^{1}, \cdots, u^{k}\right) .
$$

If $x_{k+1}^{i}$ is not a left member in the solved form of $\bar{\Sigma}_{k+1}$, the corresponding $\phi^{i}$ can be chosen arbitrarily, subject to the restrictions that (20) be satisfied and that the values

$$
\left(\frac{\partial \phi^{i}}{\partial u^{k+1}}\right)_{u^{1}=\cdots=u^{k+1}=0},
$$

taken with (18), make $J_{k+1}$ of rank $k+1$.

If we consider the solution (19) substituted in the brackets (6), let $\alpha_{1}, \cdots, \alpha_{p}$ have any values less than $k+1$, and let $\alpha_{p+1}=k+1$, we find, then, from (8), (14), and the fact that $\bar{\Sigma}_{k+1}$ is satisfied,

$$
\begin{aligned}
\frac{\partial\left[\alpha_{1} \cdots \alpha_{p}\right]}{\partial u^{k+1}}=(p & +1)\left\{\alpha_{1} \alpha_{2} \cdots \alpha_{p}(k+1)\right\}^{\prime} \\
& -(-1)^{p} \frac{\partial\left\{\alpha_{2} \cdots \alpha_{p}(k+1)\right\}}{\partial u^{\alpha_{1}}}-\cdots
\end{aligned}
$$


Whatever values the arguments in the right members of (22), other than (12) and the $u$ 's, may have, Cauchy's theorem states the existence of a unique set of functions (12) which satisfy (22) and reduce to zero for $u^{k+1}=0$. Since a set of zeros satisfies both conditions, we infer the identical vanishing of (12). The systems $\Sigma_{1}, \cdots, \Sigma_{k}, \bar{\Sigma}_{k+1}$ being satisfied, so also is $\Sigma_{k+1}$. Formulas (19) therefore define an integral variety, which is of dimension $k+1$ because $J_{k+1}$ is of rank $k+1$ on it.

If $k+2 \leqq \gamma$ and it is desired to obtain subsequently an integral variety passing through (19), we have in addition to restrict the initial values so that (16), (18), (21) make $M_{k+2}$ of rank $m_{k+2}$.

Starting with the case $k=1$, for which Cauchy's theorem immediately states the existence of an integral curve, we can construct, step by step, every integral variety of dimension $k \leqq \gamma$ on which each matrix $M_{\alpha},(\alpha=1,2, \cdots, k+1)$, has rank $m_{\alpha}$. Such integral varieties will be called non-singular, and all others singular.

Theorem 1. A generalized pfaffian system has at least one nonsingular integral variety $V_{k}$ of dimension $k$ if $k \leqq \gamma$. There is at least one $V_{k+1}$ through any $V_{k}$ if $k<\gamma$.

3. The Equivalent System of Partial Differential Equations. It is a matter of notation to assume that $\Sigma_{1}$ can be solved for $x_{1}^{1}, \cdots, x_{1}{ }^{m_{1}}$. Since $\bar{M}_{2}$ contains $M_{1}$, it follows from a well known theorem on rank that the derivatives $x_{2}{ }^{1}, \cdots, x_{2}{ }^{{ }^{11}}$ may be taken among those for which $\bar{\Sigma}_{2}$ is solved. Proceeding in this manner, we see that the conditions for a non-singular integral variety can be put in a form solved for

$$
x_{\alpha}{ }^{i_{\alpha}}, \quad\left(i_{\alpha}=1,2, \cdots, m_{\alpha} ; \alpha=1,2, \cdots, k\right),
$$

where $k$ does not exceed $\gamma$. The system of partial differential equations is then in regular form.* From the nature of the initial values which its solution can be given we immediately conclude that it is passive.

A subvariety of (3) is defined by formulas

$$
u^{\alpha}=u^{\alpha}\left(v^{1}, \cdots, v^{l}\right) .
$$

* J. M. Thomas, Proceedings National Academy of Sciences, vol. 19 (1933), pp. $451-453$. 
The brackets for the subvariety are given by

$$
\left[\beta_{1} \beta_{2} \cdots \beta_{p}\right]_{v}=\left[\alpha_{1} \alpha_{2} \cdots \alpha_{p}\right] u_{\beta_{1}}^{\alpha_{1}} u_{\beta_{2}}^{\alpha_{2}} \cdots u_{\beta_{p}}^{\alpha_{p}},
$$

where $u_{\beta}{ }^{\alpha}=\partial u^{\alpha} / \partial v^{\beta}$. From these equations we see that every subvariety of an integral variety is integral and therefore satisfies the same system of partial differential equations as the variety. From Theorem 1 every non-singular integral variety is contained in some non-singular integral variety of dimension $\gamma$. Making $k=\gamma$ in (23), we therefore get the following theorem.

THEOREM 2. The non-singular integral varieties of a generalized pfaffian system satisfy a system of partial differential equations of the first order which can be written in passive, regular form.

As a consequence, the problem of finding the non-singular integral varieties for (1) leads at once to a system of partial differential equations

$$
\bar{\Sigma}_{1}+\bar{\Sigma}_{2}+\cdots+\bar{\Sigma}_{\gamma}
$$

written in a form which is included, as was proved above, in Riquier's more general passive orthonomic form. It is in this sense that Theorem 1, and therefore Cartan's theorem, is equivalent to a special case of Riquier's theorem.

Every integral variety of (1) satisfies at least one of the systems

$$
\Sigma_{1}, \Sigma_{1}+\Sigma_{2}, \cdots, \Sigma_{1}+\Sigma_{2}+\cdots+\Sigma_{n}
$$

Conversely, a solution of any one of systems (26) defines an integral variety of (1). Riquier's method of reduction to passive orthonomic form can be applied to each system in (26), and (1) is completely solved in this manner. Riquier's method of testing whether a system has solutions must therefore be regarded as more general than Cartan's since the latter only furnishes the non-singular varieties.*

DuKe UnIVERSITY

* Compare Goursat's surmise, loc. cit., p. 381 . 